\title{
Rendimiento de la colangiografía por resonancia magnética en el diagnóstico de coledocolitiasis
}

\author{
Enrique Norero' ${ }^{1}$, Blanca N orero ${ }^{1}$, Álvaro Huete ${ }^{2}$, \\ Fernando Pimentel ${ }^{1}$, Francisco $\mathrm{Cruz}^{2}$, Luis Ibáñez ${ }^{1}$, \\ Jorge Martínez ${ }^{1}$ Nicolás Jarufe ${ }^{1}$. \\ Accuracy of magnetic resonance
cholangiopancreatography for the
diagnosis of common bile duct stones
}

Background: Magnetic resonance cholangiopancreatography (MRCP) is a non-invasive diagnostic method for choledocholithiasis. Aim: To evaluate the results of MRCP in the diagnosis of choledocholithiasis. Patients and methods: Retrospective review of MRCP reports performed between October 2001 and December 2004. We included patients with suspected choledocholithiasis who were studied with MRCP and some other confirmatory test such as endoscopic retrograde cholangiopancreatography (ERCP), surgical common bile duct exploration or transcystic colangiography (TC). Results: One hundred and twenty five patients aged $58 \pm 20$ years (70 females) were included. In 54 patients (43\%) we compared the results of MRCP with the findings of surgical common bile duct exploration or TC and in 71 (57\%) we did so with ERCP. MRCP suggested choledocholithiasis in 93 patients and in 32 it was negative. Eighty six (67\%) patients had choledocholithiasis according to TC or ERCP, including 3 patients who had a negative MRCP. Therefore the sensibility was $97 \%$, specificity $74 \%$ positive predictive value $89 \%$, negative predictive value $90 \%$ and accuracy of MRCP was $90 \%$ for the diagnosis of choledocholithiasis. Conclusions: MRCP has a high accuracy for the diagnosis of choledocholithiasis (Rev Méd Chile 2008; 136: 600-5).

(Key words: Cholangiopancreatography, endoscopic retrograde; Cholangiopancreatography, magnetic resonance; Choledocholithiasis)

Recibido el 22 de agosto, 2007. Aceptado el 12 de diciembre, 2007.

${ }^{1}$ Departamento de Cirugía Digestiva, División de Cirugía, 2Departamento de Radiología, Facultad de Medicina, Pontificia Universidad Católica de Chile. Santiago de Chile.

Se estima que entre 7\% y $16 \%$ de los pacientes Scon colelitiasis son portadores también de una coledocolitiasis $^{1,2}$. Si bien la ecografía abdominal

Correspondencia a: Dr. Nicolás Jarufe. Departamento de Cirugía Digestiva, Facultad de Medicina, Pontificia Universidad Católica de Chile. Marcoleta 367, Casilla 114-D, Santiago. Fono: 3543870. Fax: 6329620. E mail: njarufe@med.puc.cl es el estándar para el diagnóstico de colelitiasis, el diagnóstico preoperatorio de coledocolitiasis frecuentemente es difícil de establecer ${ }^{3}$.

Con la introducción de la colecistectomía laparoscópica ${ }^{4,5}$, surgió un gran interés en el diagnóstico preoperatorio de coledocolitiasis. Se han descrito varios factores de riesgo, tanto clínicos, como ictericia, coluria intermitente o colangitis; como de laboratorio: elevación de las 
fosfatasas alcalinas, de la gamaglutamiltranspeptidasa (GGT) e hiperbilimubinemia; y también ecográficos, como dilatación de la vía biliar, colelitiasis con cálculos pequeños y cálculos visibles en la vía biliar ${ }^{2,6-8}$. Sin embargo, ninguno de ellos permite confirmar o descartar definitivamente una coledocolitiasis. De esta manera, para el diagnóstico preoperatorio de coledocolitiasis, el principal método utilizado durante los últimos años ha sido la colangio-pancreatografía retrógrada endoscópica (CPRE), cuya eficacia está demostrada, pero es un método invasivo que se asocia con morbilidad y mortalidad y tiene costo elevado ${ }^{9}$.

La colangiografía por resonancia magnética (CRM) se introdujo en el año $1991^{10} \mathrm{y}$ durante los últimos años la calidad de las imágenes ha mejorado en forma significativa ${ }^{11}$. En el Hospital Clínico de la Universidad Católica se dispone de este método diagnóstico desde agosto de 2001. Sus principales ventajas son ser un método no invasivo, sin morbilidad ni mortalidad asociada. En nuestro medio, hay escasos estudios que evalúan este método ${ }^{12}$.

El objetivo de este trabajo es evaluar los resultados de la CRM para el diagnóstico de la coledocolitiasis.

\section{MATERIAL Y MÉTODO}

Estudio retrospectivo a partir de los informes de las CRM realizadas en el Servicio de Radiología de nuestro hospital, entre octubre de 2001 y diciembre de 2004. Se incluyó a los pacientes con una CRM por sospecha de coledocolitiasis, ictericia obstructiva, pancreatitis y colangitis, que tuvieron algún examen confirmatorio para coledocolitiasis, ya sea CPRE, colangiografía intraoperatoria (CIO) o exploración quirúrgica de la vía biliar.

Se excluyeron del estudio los pacientes con diagnóstico previo de fístula biliar, estenosis 0 lesión de la vía biliar, disfunción del esfínter de Oddi y cáncer de páncreas, vías biliares, vesícula o metástasis de otros tumores.

En los pacientes incluidos, se revisó los registros de la CPRE del laboratorio de endoscopia y los protocolos quirúrgicos de la base de datos informatizada del Servicio de Cirugía.

Se consideró diagnóstico confirmado de coledocolitiasis en la CPRE o CIO, a la presencia de una imagen negativa característica con el contraste, o su visualización directa por vía endoscópica o en la exploración quirúrgica.

Se recogieron los datos demográficos de la serie y el tiempo transcurrido, en días, entre la CRM y el examen confirmatorio.

Los datos con variables continuas se expresaron en promedios \pm desviación estándar.

Con los resultados de la CRM y de los exámenes confirmatorios se calcularon la sensibilidad, la especificidad, el valor predictivo positivo, el valor predictivo negativo, la razón de probabilidad positiva y negativa ("likelihood ratio", LR) y la exactitud diagnóstica.

Todas las CRM se realizaron con un equipo Philips 1.5 modelo Intera con sistema $1.5 \mathrm{~T}$, utilizando gradientes de alto rendimiento $(30 \mathrm{mT} / \mathrm{m})$, bobinas de superficie de 4 canales y secuencias ponderadas en T2 con técnica de «disparo único» con cortes finos multiplanares $(3-5 \mathrm{~mm})$ y proyecciones colangiográficas gruesas $(4-6 \mathrm{~cm})$. El resultado del examen fue informado por uno de los dos radiólogos especializados en CRM.

\section{RESULTADOS}

Durante los tres años del estudio se realizaron 908 CRM. Se incluyeron en este trabajo 125 pacientes en quienes el examen se realizó por sospecha de coledocolitiasis y se contó con algún estudio confirmatorio. Este grupo está compuesto por 70 (56\%) mujeres y 55 (44\%) hombres, con una edad de $58 \pm 20$ años.

Las indicaciones para realizar la CRM se describen en la Tabla 1. Los exámenes confirmatorios se

\section{Tabla 1. Indicaciones de la CRM en este estudio}

\begin{tabular}{|lrr|}
\hline Indicación & $\mathrm{n}$ & $\%$ \\
\hline Ictericia obstructiva & 30 & 24 \\
Colangitis & 6 & 5 \\
Pancreatitis & 17 & 14 \\
Alteración del laboratorio & 72 & 57 \\
y ecografía & 72 & \\
\hline
\end{tabular}

CRM: Colangiografía por resonancia magnética. 
describen en la Tabla 2. El tiempo promedio entre la CRM y el examen confirmatorio fue de $2 \pm 5$ días para la CIO y de $3 \pm 18$ días para la CPRE.

La CRM detectó una coledocolitiasis en 93 de los 125 pacientes, en 83 de éstos se confirmó la coledocolitiasis por CPRE o CIO; los 10 restantes fueron considerados falsos positivos. En 32 pacientes la CRM fue negativa para coledocolitiasis. En 3 de ellos la CPRE (2 pacientes) o la CIO (1 paciente) encontraron una coledocolitiasis, estos pacientes fueron considerados falsos negativos. $\mathrm{Al}$ comparar los resultados con la CPRE o la CIO, se calculó que para el diagnóstico de coledocolitiasis la CRM tuvo una sensibilidad de $97 \%$, una especificidad de $74 \%$, con un valor predictivo positivo de $89 \%$, y un valor predictivo negativo de $90 \%$. Con un LR positivo de 3,7, un LR negativo de 0,04 y una exactitud diagnóstica de $90 \%$ (Tabla 3 ).

\section{Discusión}

Con la introducción de la CRM como nueva herramienta diagnóstica no invasiva en el diagnóstico de coledocolitiasis, se eliminan los riesgos inherentes de los otros métodos diagnósticos. Cabe recordar que con la CPRE se describe una morbilidad de $9 \%$ y una mortalidad de 0,4\% ${ }^{9}$. Además, existen pacientes en los cuales es más difícil o imposible realizar una CPRE, ya sea por la presencia de un divertículo duodenal grande, 0 por el antecedente de operaciones con una derivación con asa desfuncionalizada en $\mathrm{Y}$ de Roux, una reconstrucción tipo Billroth II, etc. En ellos, la CRM permite fácilmente la visualización
Tabla 2. Examen confirmatorio utilizado

\begin{tabular}{|lll|}
\hline Examen & $\mathrm{n}$ & $\%$ \\
\hline CIO & 54 & 43 \\
CPRE & 71 & 57 \\
\hline
\end{tabular}

CIO: Colangiografía intraoperatoria, CPRE: Colangio-pancreatografía retrograda endoscópica.

de la vía biliar ${ }^{13}$, lo que antes era sólo posible con la colangiografía percutánea o la colangiografía endovenosa. La CRM no requiere medio de contraste, lo que la convierte en una buena alternativa en pacientes con alergia al yodo ${ }^{11}$.

Como estudio preoperatorio en un paciente portador de colelitiasis, con factores de riesgo de coledocolitiasis, la CRM es una buena herramienta frente a la CIO. Esta última, si bien es bastante segura, no está completamente exenta de complicaciones, describiéndose lesiones de la vía biliar por excesiva manipulación en $0,4 \%$ de los pacientes y además en $3 \%$ de los casos no es posible realizarla, principalmente por dificultad en la canulación del conducto cístico ${ }^{14}$.

La CRM no sólo permite conocer la presencia o ausencia de coledocolitiasis, sino que, al producir imágenes del árbol biliar similares a las logradas por métodos radiológicos directos, visualiza también el diámetro de la vía biliar, el número de cálculos, su ubicación, detalles de la vía biliar intrahepática, variantes anatómicas; todos ellos importantes en el momento de planificar el tratamiento (Figura 1). También se puede asociar a la

Tabla 3. Tabla de contingencia con los resultados de CRM y examen confirmatorio

\begin{tabular}{|cl|ccc|}
\hline \multicolumn{4}{|c|}{ Ex. confirmatorio (CIO-ERCP) } \\
\hline & & $(+)$ & $(-)$ & Total \\
& $(+)$ & 83 & 10 & 93 \\
CRM & $(-)$ & 3 & 29 & 32 \\
& Total & 86 & 39 & 125 \\
\hline
\end{tabular}

Sensibilidad 97\%; especificidad 74\%; valor predictivo positivo $89 \%$; valor predictivo negativo $90 \%$; "likelihood ratio" positiva 3,7, "likelihood ratio" negativa 0,04, exactitud 90\%. 


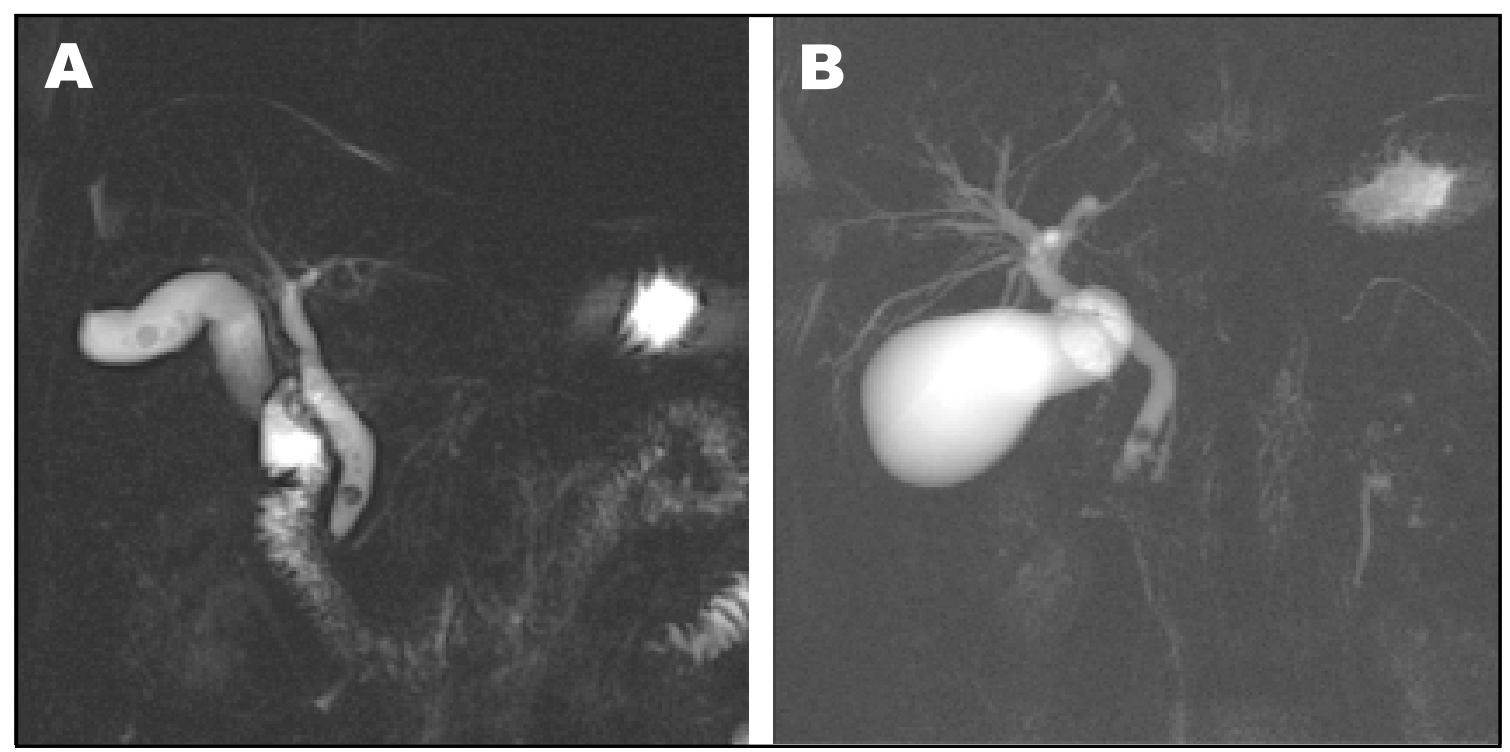

Figura 1 (a y b). Imágenes de colangiografía por resonancia magnética en 2 pacientes distintos en quienes se confirmó una coledocolitiasis.

CRM una resonancia nuclear de abdomen en los casos de sospecha de patología hepática o pancreática.

Una desventaja de la CRM es que es un método puramente diagnóstico y no permite la visualización directa de la ampolla de Vater, ni la toma de biopsias como con la $\mathrm{CPRE}^{11}$. Por otro lado, hay algunas situaciones como artefactos metálicos (clips quirúrgicos), pneumobilia y papila prominente, que disminuyen la exactitud diagnóstica de la CRM para la coledocolitiasis ${ }^{15}$. Inicialmente, otra desventaja de este método fue su baja disponibilidad en el medio nacional; esta situación ha cambiado y actualmente se realiza en muchos centros del país.

Actualmente existe una controversia sobre cuál es el mejor método de tratamiento de la litiasis de la vía biliar asociada a la colelitiasis: el tratamiento endoscópico previo a la colecistectomía o la cirugía clásica o laparoscópica de la vía biliar, en un tiempo, con la extracción de la vesícula16,17. Esta decisión depende en gran medida del grupo tratante, de los recursos del centro y de las características de cada paciente ${ }^{2,18}$. En este contexto, la CRM aporta una información valiosa en la planificación del tratamiento. Ante la presencia de una panlitiasis, con cálculos mayores de $2 \mathrm{~cm}$ y estenosis de la vía biliar, la estrategia aconsejable sería la exploración por laparotomía clásica.

Con el desarrollo de la cirugía laparoscópica de la vía biliar, se ha hecho fundamental tener información sobre la ubicación de los cálculos, su relación a la desembocadura del conducto cístico, si están impactados, el tamaño de los cálculos y el diámetro de la vía biliar. Cuando no se cumplen criterios previamente establecidos los pacientes no serán buenos candidatos a este abordaje ${ }^{19,20}$. El tener esta información en el preoperatorio permite una mejor planificación del equipo quirúrgico y del instrumental necesario en pabellón: coledocoscopio, canastillo de Dormia, balones de Fogarty, etc ${ }^{20}$.

Previo a la introducción de la CRM, la decisión de estudiar la vía biliar dependía de los factores de riesgo de coledocolitiasis. El valor predictivo de éstos es aún limitado y la mayońa de los pacientes con uno o dos factores de riesgo no tenían coledocolitiasis, y cierta proporción de ellos fueron sometidos a un procedimiento innecesario, CIO, exploración quinúrgica de la vía biliar o $\mathrm{CPRE}^{6,7}$. En pacientes con colelitiasis sin factores de riesgo para coledocolitiasis, no parece justificada la realización de una CRM en el preoperatorio, a pesar de que se han descrito hasta $6 \%$ de coledocolitiasis asociada en estos pacientes ${ }^{6}$. Nos 
parece que los pacientes con colelitiasis y uno o dos factores de riesgo clásicos para coledocolitiasis son los que tienen la mejor indicación de una CRM preoperatoria. Actualmente, en pacientes con 3 o más factores de riesgo de coledocolitiasis también realizamos una CRM y según los resultados de ella, decidimos la necesidad de exploración de la vía biliar, ya sea mediante CPRE o por cirugía.

Creemos que al introducir la CRM en la práctica clínica en pacientes con sospecha de coledocolitiasis, la proporción de CPRE que son sólo diagnósticas debería reducirse, y así también el número de pacientes con complicaciones derivadas del procedimiento. Este aspecto actualmente es motivo de un estudio en nuestro centro.

\section{REFERENCIAS}

1. Perissat J, Huibregtse $K$, Keane F, Russell $R$, NeoptoLEMOS J. Managment of bile duct stones in the era of laparoscopic cholecystectomy. Br J Surg 1994; 81: 799-810.

2. IbÁÑez L, López F, Guzmán S, Hamlton J, Arroyo C, Carcomo C et al. Factores de riesgo de coledocolitiasis. Rev Chil Cir 1998; 50: 544-6.

3. Pumarino A, Humeres $R$, Ríos $H$, Díaz $R$, Agar $S$, SuÁREZ L ET AL. Determinación de variables asociadas a coledocolitiasis y su valor predictivo. Rev Chil Cir 1996; 48: 587-90.

4. Reynolds W. The first laparoscopic cholecystectomy. JSLS 2001; 5: 89-94.

5. MüHE E. Die erste Cholecystektomie durch das Laparaskop. Langenbecks Arch Chir 1986; 369: 804-6.

6. Burmeister R, Apablaza S, Soto D. Coledocolitiasis en el Hospital Clínico San Borja Arriarán: estudio prospectivo. Rev Chil Cir 2002; 54; 148-52.

7. Abboud P, Malet P, Berlin J, Staroscik R, Cabana M, Clarke J et al. Predictors of common bile duct stones prior to cholecystectomy: a meta-analysis. Gastrointest Endosc 1996; 44: 450-5.

8. Benavides C, Jarufe N, Burmeister R, Ricaurte F, Saxton F, Marro P ET aL. Factores de riesgo de coledocolitiasis. Rev Chil Cir 1999; 51: 483-6.

9. Freeman M, Nelson D, Sherman S, Haber G, Herman M, Dorsher P ET AL. Complications of endoscopic biliary sphincterotomy. N Engl J Med 1996; 335: 909-18.
En resumen, este trabajo confirma los buenos resultados de la CRM en el diagnóstico de coledocolitiasis, con una exactitud diagnóstica de $90 \% 21$ 25 y pocos falsos negativos, si bien presenta algunas limitaciones al tratarse de un estudio retrospectivo, donde se tuvo un examen confirmatorio sólo en un grupo limitado de pacientes. Por otro lado, el lapso promedio de 2 ó 3 días entre la CRM y el examen de referencia podría cambiar los resultados obtenidos, debido a los conocidos fenómenos de migración espontánea de los cálculos de la vía biliar al intestino ${ }^{26}$. Si bien la más exacta validación del método requiere de un estudio prospectivo, esta evaluación nos acerca a la utilidad del mismo en nuestra práctica clínica.

10. WALLNER BK, Schumacher KA, WeidenMaier W, FrieDRICH JM. Dilated biliary tract: evaluation with MR cholangiography with a T2-weighted contrastenhanced fast sequence. Radiology 1991; 181: 805-8.

11. BARISH M, YuCEL K, FERRUCCI J. Magnetic resonance cholangiopancreatography. N Engl J Med 1999; 341: 258-64.

12. Busel D, Pérez L, Arroyo A, Ortega D, Neidmann J, Palavecino P et al. Colangiorresonancia vs ultrasonido focalizado en pacientes con ictericia 0 sospecha de obstrucción de la vía biliar. Resultados preliminares. Rev Chil Radiol 2003; 9: 173-81.

13. Soto J, Yucel E, Barish A, Chutani R, Ferrucci J. MR Cholangiopancreatography after unsuccessful or incomplete ERCP. Radiology 1996; 199: 91-8.

14. Csendes A, Burdiles P, Díaz J, Maluenda F, Korn O, RoJAs J. Estudio prospectivo sobre la prevalencia de coledocolitiasis de acuerdo a la presencia de factores de riesgo. Análisis de 464 colangiografías operatorias. Rev Chil Cir 1998; 50: 385-93.

15. David V, Reinhold C, Hochman M, Chutani R, Mckee $\mathrm{J}$, WAXMAN I ET AL. Pitfalls in the interpretetion of MR cholangiopancreatography. AJR 1998; 170: 1055-9.

16. Tranter S, Thompson M. Comparison of endoscopic sphincterotomy and laparoscopic exploration of the common bile duct. Br J Surg 2002; 89: 1495-504.

17. Diagnosis and treatment of common bile duct stones (CBDS). Results of a concensus development conference. Scientific Committee of the 
European Association for Endoscopic Surgery (E.A.E.S.). Surg Endosc 1998; 12: 856-64.

18. Pérez G, Escalona A, Jarufe N, Ibáñez L, Viviani P, García C et al. Prospective randomized study of T-tube versus biliary stent for common bile duct decompression after open choledocotomy. World J Surg 2005; 29: 869-72.

19. Pérez G, Pimentel F, Ibáñez L. Exploración de la vía biliar por coledocotomía laparoscópica. Rev Chil Cir 2002; 54: 251-5.

20. Berthou J, Drouard F, Charbonneau P, Moussaler K. Evaluation of laparoscopic management of common bile duct stones in 220 patients. Surg Endosc 1998; 12: 16-22.

21. Romagnuolo J, Bardou M, Rahme E, Joseph L, Reinhold C, Barkun A. Magnetic resonance cholangiopancreatography: a meta-analysis of test performance in suspected biliary disease. Ann Intern Med 2003; 139: 547-57.
22. Barish MA, Yucel EK, Soto JA, Chuttani R, Ferrucci JT. MR cholangiopancreatography: efficacy of three-dimensional turbo spin-echo technique. AJR 1995; 165: 295-300.

23. Guibaud L, Bret PM, Reinhold C, Atri M, Barkun AN. Diagnosis of choledocholithiasis: value of MR cholangiography. AJR 1994; 163: 847-50.

24. Takehara $\mathrm{Y}$, Ichijo $\mathrm{K}$, ToOyama $\mathrm{N}$, Kodaira $\mathrm{N}$, Yamamoto $\mathrm{H}$, Tatami M ET AL. Breath-hold MR cholangiopancretography with a long-echo-train fase spin-echo sequence and a surface coil in chronic pancreatitis. Radiology 1994; 192: 73-8.

25. Guibaud L, Bret PM, Reinhold C, Atri M, Barkun AN. Bile duct obstruction and choledocholitiasis: diagnosis with MR cholangiography. Radiology 1995; 197: 109-15.

26. Acosta J, Ledesma C. Gallstone migration as a cause of acute pancreatitis N Engl J Med 1974; 290: 484-7. 